\title{
Protocolo do Hospital Universitário Walter Cantídio para avaliação de cardiotoxicidade em pacientes submetidos à quimioterapia
}

\section{Walter Cantídio University Hospital's protocol for evaluation of cardiotoxicity in patients submitted to chemotherapy}

Márcia Beatriz de Jesus Lima․ Ana Gardênia Liberato Ponte Farias². Danielle Melo de Leopoldino ${ }^{3}$.

1 Médica residente do Programa de Ecocardiografia, Universidade Federal do Ceará (UFC), Fortaleza, Ceará, Brasil. 2 Doutora em Cardiologia, Médica preceptora do Programa de Ecocardiografia pela Universidade Federal do Ceará (UFC), Fortaleza, Ceará, Brasil. 3 Mestra em Ciências Médicas, supervisora do Programa de Ecocardiografia pela Universidade Federal do Ceará (UFC), Fortaleza, Ceará, Brasil.

\section{RESUMO}

O câncer é um problema de saúde pública. Apesar disso, é inegável que novas ferramentas diagnósticas em combinação com novas terapias, regimes de tratamento mais intensivos e melhores cuidados de suporte aumentaram a expectativa de vida dos pacientes portadores dessa enfermidade. Com o aumento da taxa de sobrevida nesses pacientes, houve um aumento, em paralelo, dos efeitos cardíacos adversos provocados pelas novas terapias. Atualmente, observa-se uma mudança no paradigma em relação ao prognóstico do paciente oncológico, que passa a ser visto como um portador de uma doença crônica. O presente protocolo tem como objetivo reduzir os riscos da cardiotoxicidade do tratamento quimioterápico, bem como disseminar recomendações práticas para a monitorização da função cardiovascular antes, durante e após o tratamento do paciente. Através da realização de ecodopplercardiogramas com strain seriados e dosagem de troponina antes e após os ciclos quimioterápicos, os pacientes serão seguidos, conjuntamente, no ambulatório de cardiologia e no serviço de oncologia para avaliação e tomada de decisões clínicas, no intuito de prevenir e oferecer tratamento precoce à cardiotoxicidade induzida por quimioterápicos, de acordo com fluxograma desenvolvido.

Palavras-chave: Câncer. Cardiotoxicidade. Ecocardiografia. Torção mecânica.

\section{ABSTRACT}

Cancer is a public health problem. Despite this, it is undeniable that new diagnostic tools in combination with new therapies, more intensive treatment regimens and better supportive care have increased the life expectancy of patients with this disease. With the increase in the survival rate in these patients, there was a parallel increase in the adverse cardiac effects caused by the new therapies. Currently, there is a change in the paradigm regarding the prognosis of the cancer patient, who is now seen as a carrier of a chronic disease. The present protocol aims to reduce the risks of cardiotoxicity of the chemotherapy treatment, as well as to disseminate practical recommendations for the monitoring of cardiovascular function before, during and after the treatment of the patient. Through the accomplishment of series-based Doppler echocardiograms with strain and troponin dosing before and after the chemotherapy cycles, the patients will be followed together in the cardiology outpatient clinic and the oncology department to evaluate and make clinical decisions in order to prevent and offer treatment to the cardiotoxicity induced by chemotherapy, according to the developed flowchart.

Keywords: Cancer. Cardiotoxicity. Echocardiography. Mechanical torsion.

Autor correspondente: Márcia Beatriz de Jesus Lima, Rua Waldery Uchoa, 600, Benfica, Fortaleza, Ceará. CEP: 60020-100. Telefone: +55 85 99805-7474. E-mail: marciabiatriz@hotmail.com

Conflito de interesses: Não há qualquer conflito de interesses por parte de qualquer um dos autores.

Recebido em: 02 Fev 2017; Revisado em: 24 Set 2017; Aceito em: 24 Set 2017. 


\section{INTRODUÇÃO}

O câncer é um problema de saúde pública. Apesar disso, é inegável que novas ferramentas diagnósticas em combinação com novas terapias, regimes de tratamento mais intensivos e melhores cuidados de suporte aumentaram a expectativa de vida dos pacientes portadores dessa enfermidade. ${ }^{1}$ Com o aumento da taxa de sobrevida nesses pacientes, houve um aumento, em paralelo, dos efeitos cardíacos adversos provocados pelas novas terapias. ${ }^{2}$ Em 1973, Edward Lefrak foi o primeiro a descrever uma forma grave de insuficiência cardíaca irreversível em doentes tratados com antraciclinas; ${ }^{2}$ desde então, pesquisadores têm buscado soluções para evitar o que mais tarde ficou conhecido como cardiotoxicidade induzida pelos quimioterápicos.

Atualmente, observa-se uma mudança no paradigma em relação ao prognóstico do paciente oncológico, que passa a ser visto como um portador de uma doença crônica, com real possibilidade de, ao longo de sua evolução, apresentar descompensações agudas, como as manifestações cardiovasculares. Nesse novo contexto, mais da metade dos pacientes expostos à antraciclina poderão desenvolver disfunção cardíaca detectável no período compreendido entre 10 e 20 anos, após a quimioterapia; e 5\% deles poderão evoluir para insuficiência cardíaca (IC) evidente. Diante de tais estatísticas, a fração de ejeção do ventrículo esquerdo (FEVE) pode não ser suficientemente sensível para identificar a cardiotoxicidade induzida pelos quimioterápicos. ${ }^{3}$

Assim, os pacientes submetidos a tratamento quimioterápico devem ser rastreados de forma diferenciada em relação a sintomas cardiovasculares. História clínica, exame físico, métodos de imagem (como o ecodopplercardiograma) e biomarcadores (como a troponina) são essenciais nesse seguimento. ${ }^{4}$

O presente protocolo tem como objetivo reduzir os riscos da cardiotoxicidade do tratamento quimioterápico, bem como disseminar recomendações práticas para a monitorização da função cardiovascular antes, durante e após o tratamento do paciente.

\section{DEFINIÇÃO DE CARDIOTOXICIDADE}

Nas últimas duas décadas, as definições de cardiotoxicidade nos ensaios clínicos de oncologia são baseadas nas medidas da fração de ejeção do ventrículo esquerdo (FEVE). Nos Estados Unidos, o Instituto Nacional de Saúde (NIH), seguindo esse padrão, define cardiotoxicidade, classificando-a em graus da seguinte maneira: ${ }^{3-5}$

Grau I: redução assintomática da FEVE entre 10\% e $20 \%$;

Grau II: redução da FEVE para baixo de $20 \%$ do basal ou para baixo do normal (considerado o quadro específico de cada paciente);

Grau III: insuficiência cardíaca sintomática.
Outra definição (e uma das mais acuradas) de cardiotoxicidade é a formulada pelo Comitê de Revisão e Avaliação dos Estudos Clínicos de Trastuzumabe, ${ }^{5}$ segundo a qual, a cardiotoxicidade associada a quimioterápicos pode apresentar-se como uma das seguintes formas clínicas:

1) miocardiopatia com redução da fração de ejeção ventricular esquerda (FEVE), quer seja global, quer, segmentar, acometendo mais gravemente o septo interventricular;

2) sintomas associados à IC;

3) sinais associados à IC, tais como B3 e/ou taquicardia;

4) redução na FEVE, em comparação à basal, de pelo menos $5 \%$ (ou de modo que fique inferior a $55 \%$ - valor estabelecido como limiar de normalidade), com sinais ou sintomas de IC concomitantes; ou, ainda, redução na FEVE na faixa de pelo menos $10 \%$ (ou de modo que fique inferior a $55 \%$ - valor estabelecido como limiar de normalidade), sem sinais ou sintomas concomitantes.

Partindo do conceito formulado pelo Comitê, qualquer um dos quatro critérios é suficiente para confirmar o diagnóstico de cardiotoxicidade.

Embora ambos os conceitos apresentados tenham se baseado no método científico para suas respectivas elaborações, ainda não existe um consenso sobre a definição ideal de cardiotoxicidade induzida por quimioterápicos. A despeito disso, as duas modalidades de definição se revelam servíveis para nortear estudos na área.

Independente da definição inicial adotada, em suas ocorrências, a cardiotoxicidade pode apresentar-se de forma aguda, subaguda ou crônica. A cardiotoxicidade aguda ou subaguda caracteriza-se por alterações súbitas na repolarização ventricular, alterações no intervalo QT, arritmias supraventriculares e ventriculares, síndromes coronarianas agudas, pericardite e miocardite, geralmente observadas desde o início até 14 dias após o término do tratamento.

A cardiotoxicidade crônica, por sua vez, pode ser diferenciada em dois tipos, de acordo com o início dos sintomas clínicos. O primeiro subtipo ocorre dentro do período de um ano, contado do término da quimioterapia; já o segundo, ocorre geralmente após esse um ano do término da quimioterapia.

A manifestação mais típica de cardiotoxicidade crônica é a disfunção ventricular sistólica ou diastólica que pode levar o paciente de insuficiência cardíaca congestiva até morte cardiovascular. ${ }^{3-5}$ Nesse contexto, a incidência de disfunção ventricular sintomática ou assintomática nas séries varia entre 5 e 30\%, sendo que pacientes com disfunção ventricular prévia, hipertensão arterial, diabetes mellitus, associação de quimioterápicos, irradiação mediastinal e suscetibilidade genética apresentam risco mais elevado para esta complicação, como apresentado no Quadro 1.6 
Quadro 1. Fatores de risco relacionados ao potencial efeito cardiotóxico.

Idade (<15 anos e $>70$ anos)
Sexo feminino
Radioterapia prévia
Associação de quimioterápicos
História prévia de doença cardíaca
Diabetes
Obesidade
Susceptibilidade individual à toxicidade do fármaco, que é
dose-dependente
Raça (negro > branco)
Mutação HFE (hemocromatose)

Fonte: Kalil R Filho, Hajjar LA, Bacal F, Hoff PM, Diz M del $\mathrm{P}$, Galas FR, et al. I Diretriz Brasileira de Cardio-Oncologia da Sociedade Brasileira de Cardiologia. Arq Bras Cardiol. 2011;96(2 supl.1):5.

\section{INSUFICIÊNCIA CARDÍACA}

Uma das principais e mais temidas complicações do tratamento quimioterápico é a insuficiência cardíaca (IC). ${ }^{7}$ De acordo com diretrizes nacionais e internacionais, tal insuficiência é uma síndrome clínica complexa de caráter sistêmico, definida como disfunção cardíaca que ocasiona inadequado suprimento sanguíneo para atender às necessidades metabólicas tissulares. $^{8,9}$

A insuficiência cardíaca também pode ser classificada em estágios evolutivos, ${ }^{10,11}$ em cada um dos quais, como a nomenclatura perfeitamente sugere, há um avanço da enfermidade quando comparado à descrição apresentada do nível antecessor:

- Estágio A - inclui pacientes sob risco de desenvolver insuficiência cardíaca, mas ainda sem doença estrutural perceptível e sem sintomas atribuíveis à insuficiência cardíaca.

- Estágio B - pacientes que adquiriram lesão estrutural cardíaca, mas ainda sem sintomas atribuíveis à insuficiência cardíaca.

- Estágio C - pacientes com lesão estrutural cardíaca e sintomas atuais ou pregressos de insuficiência cardíaca.

- Estágio D - pacientes com sintomas refratários ao tratamento convencional e que requerem intervenções especializadas ou cuidados paliativos.

A IC pode, ainda, ser categorizada, com base na intensidade de sintomas, em 4 classes propostas pela New York Heart
Association. Esta classificação possui caráter funcional, como se vê a seguir: ${ }^{12}$

Classe I - ausência de sintomas (dispneia) durante atividades cotidianas.

Classe II - sintomas desencadeados por atividades cotidianas.

Classe III - sintomas desencadeados em atividades menos intensas que as cotidianas ou pequenos esforços.

Classe IV - sintomas em repouso.

\section{FÁRMACOS ENVOLVIDOS E FISIOPATOLOGIA}

A cardiotoxicidade crônica é o foco deste protocolo, que, desde as linhas iniciais, advoga que a detecção precoce da enfermidade, associada à instituição de medidas clínicas adequadas, constitui medida suficientemente capaz de mudar o prognóstico acerca da patologia, o que, inegavelmente, contribuiria para a melhoria da sobrevida dos pacientes.

Quando observada na modalidade crônica, a cardiotoxidade se divide em dois subgrupos, cuja análise se faz necessária. Primeiro, o tipo I resulta do uso de agentes antracíclicos, capazes de causar uma toxicidade "dose dependente"; nele, o dano celular ocorre devido a fatores genéticos individuais, bem como a uma lesão mitocondrial ou à formação de radicais livres de oxigênio, que podem exacerbar uma disfunção cardíaca pré-existente ou, a depender do paciente analisado, desencadear uma disfunção cardíaca. Essa lesão resulta em morte celular, o que acarreta um dano irreversível.

O tipo II resulta do uso de inibidores da tirosina-quinase e do trastuzumabe. O dano celular, nesse caso, ocorre por alterações transitórias nos elementos contráteis dos miócitos e a recuperação da função cardíaca é frequentemente vista após a interrupção do tratamento ${ }^{1}$, como demonstrado na Tabela 1.

Classicamente, estão bem definidos os efeitos tóxicos para os miócitos de fármacos do grupo das antraciclinas (cardiotoxicidade tipo I), que foi o primeiro estudado, sendo, por isso, arquétipo de cardiotoxicidade, embora sejam as antraciclinas muito utilizadas em vários tipos de neoplasias. ${ }^{13} \mathrm{~A}$ cardiotoxicidade das antraciclinas (doxorrubicina, epirrubicina e idarrubicina) caracteriza-se por queda na fração de ejeção do ventrículo esquerdo; ela ocorre entre 5\% e 25\% dos casos, inicia-se nas primeiras doses e está relacionada à dose cumulativa, especialmente àquelas superiores a $400 \mathrm{mg} / \mathrm{m}^{2} \mathrm{de}$ superfície corpórea, como demonstrado na Tabela 2.

Em tal dosagem, observa-se dano permanente miocárdico, caracterizado por apoptose dos miócitos, a qual resulta em fibrose e perda da função cardíaca. ${ }^{14} \mathrm{~A}$ toxicidade relacionada à ciclofosfamida, também considerada tipo I, geralmente é aguda ou subaguda, tem relação com a dose e é irreversível, na maioria dos casos. A ciclofosfamida e a ifosfamida estão relacionadas à disfunção ventricular entre $10 \%$ e $20 \%$ dos casos. $^{15}$ 
Tabela 1. Tipos de cardiotoxicidade crônica.

\begin{tabular}{|c|c|c|}
\hline & Tipo I & Tipo II \\
\hline Agente característico & Doxorrubicina & Trastuzumabe \\
\hline $\begin{array}{l}\text { Curso clínico e resposta típica a } \\
\text { terapia antirremodelamento (beta- } \\
\text { bloqueadores, IECA) }\end{array}$ & $\begin{array}{l}\text { Pode estabilizar, mas os danos } \\
\text { subjacentes parecem ser permanentes } \\
\text { e irreversíveis; recorrência em meses } \\
\text { ou anos pode estar relacionada ao } \\
\text { estresse cardíaco sequencial }\end{array}$ & $\begin{array}{l}\text { Alta probabilidade de recuperação } \\
\text { (perto do estado cardíaco basal) em } \\
2-4 \text { meses depois da interrupção } \\
\text { (reversível) }\end{array}$ \\
\hline Dose efeito & Cumulativa, dose relacionada & Não relacionada à dose \\
\hline Efeitos da reintrodução & $\begin{array}{l}\text { Alta probabilidade de disfunção } \\
\text { recorrente que é progressiva; pode } \\
\text { resultar em insuficiência cardíaca } \\
\text { intratável ou morte }\end{array}$ & $\begin{array}{l}\text { Cada vez mais evidências para a } \\
\text { segurança relativa da reintrodução } \\
\text { (dados adicionais são necessários) }\end{array}$ \\
\hline Ultraestrutura & $\begin{array}{l}\text { Vacúolos; desarranjo miofibrilar; } \\
\text { necrose }\end{array}$ & $\begin{array}{l}\text { Nenhuma anormalidade ultraestrutural } \\
\text { aparente (embora não completamente } \\
\text { estudado) }\end{array}$ \\
\hline
\end{tabular}

Fonte: Plana JC, Galderisi M, Barac A, Ewer MS, Ky B, Scherrer-Crosbie M, et al. Expert Consensus for Multimodality Imaging Evaluation of Adult Patients during and after Cancer Therapy: A Report from the American Society of Echocardiography and the European Association of Cardiovascular Imaging. J Am Soc Echocardiogr. 2014;27(9)913.

Tabela 2. Principais agentes quimioterápicos utilizados no tratamento do câncer e a incidência de cardiotoxicidade (redução da fração de ejeção e/ou insuficiência cardíaca).

\begin{tabular}{lcc}
\hline Agente quimioterápico & $\begin{array}{l}\text { Incidência (\%) de disfunção ventricular ou } \\
\text { insuficiência cardíaca }\end{array}$ & Frequência de uso \\
\hline $\begin{array}{l}\text { Antraciclinas (doxorrubicina, epirrubicina, } \\
\text { idarrubicina) }\end{array}$ & $5 \%$ a $35 \%$ dos casos $\left(\right.$ dose acima de $\left.400 \mathrm{mg} / \mathrm{m}^{2}\right)$ & ++++ \\
$\begin{array}{l}\text { Agentes alquilantes (ciclofosfamida, } \\
\text { ifosfamida) }\end{array}$ & $2 \%$ a $10 \%$ dos casos & ++ \\
$\begin{array}{l}\text { Agentes antimicrotúbulos (docetaxel, } \\
\text { paclitaxel) }\end{array}$ & $2 \%$ a $10 \%$ dos casos & ++ \\
$\begin{array}{l}\text { Anticorpos monoclonais e inibidores da tirosina-quinase } \\
\text { Trastuzumabe }\end{array}$ & $2 \%$ a $28 \%$ dos casos & ++ \\
Bevacizumabe & $2 \%$ a $10 \%$ dos casos & ++ \\
Sunitinibe & $3 \%$ a $10 \%$ dos casos & ++ \\
\hline
\end{tabular}

Fonte: Kalil R Filho, Hajjar LA, Bacal F, Hoff PM, Diz M del P, Galas FR, et al. I Diretriz Brasileira de Cardio-Oncologia da Sociedade Brasileira de Cardiologia. Arq Bras Cardiol. 2011;96(2 supl.1):4.

O segundo grupo em importância como causa de toxicidade tem como representantes o trastuzumabe e o bevacizumabe (cardiotoxicidade tipo II). O trastuzumabe causa disfunção ventricular em até $28 \%$ dos casos. Com essa droga, ocorre disfunção transitória reversível dos miócitos sem que haja relação com a dose, resultando em melhor prognóstico. ${ }^{16} \mathrm{O}$ sunitinibe e o bevacizumabe, quando observados, determinam cardiotoxicidade em $3 \%$ a $12 \%$ dos casos. ${ }^{3}$

Merece destaque a constatação pela dosagem de biomarcadores como troponina, em que a agressão miocárdica com os diferentes quimioterápicos ocorre precocemente e com maior frequência do que aquela reportada pela análise de fração de ejeção reduzida, sinalizando a ocorrência de cardiotoxicidade.

\section{ECOCARDIOGRAFIA E BIOMARCADORES}

Os métodos utilizados para o diagnóstico da cardiotoxicidade são baseados, principalmente, na queda da fração de ejeção do ventrículo esquerdo (FEVE). A fração de ejeção é comumente aceita como medida da função sistólica cardíaca e é aceita como indicador de prognóstico em paciente com 
insuficiência cardíaca. ${ }^{1,17}$ Classicamente, a toxicidade induzida por quimioterápicos é defina por uma queda na fração de ejeção, mas, mesmo em indivíduos saudáveis, a FEVE sofre uma grande variação; então, a FEVE indicativa de dano ventricular só pode ser apropriadamente definida quando comparações são feitas entre um estudo basal do paciente e exames subsequentes. Embora uma queda acentuada na fração de ejeção durante ou após o tratamento de quimioterapia esteja associado com insuficiência cardíaca sintomática, existe uma considerável discussão sobre qual grau de queda na fração de ejeção constitui cardiotoxicidade. ${ }^{10}$

Vários métodos de imagem podem ser utilizados no seguimento clínico desses pacientes, como a ressonância nuclear magnética e a ventriculografia radioisotópica; porém, a ecocardiografia tem sido preferencial nesse seguimento, por ser um método de imagem não invasivo e que, por essa razão, não expõe o paciente à radiação ionizante. Tal exame, quando comparado aos demais no aspecto custo-benefício, apresenta vantagens em razão de poder ser repetido sem grandes ônus, se necessário, sendo, por isso, amplamente difundido e facilmente reprodutível; além de fornecer informações adicionais sobre as valvas e a função diastólica ventricular. ${ }^{18}$

Apesar do uso e da ampla aceitação da FEVE como medida de cardiotoxicidade, existem evidências crescentes indicando que a fração de ejeção não é um marcador sensível de injúria miocárdica. A hipótese é de que mudanças na fração de ejeção só ocorrem tardiamente no processo patológico, depois que os mecanismos compensatórios, como a reserva contrátil miocárdica e outras vias, estão esgotados..$^{18}$ Outrossim, um dos desafios chave na monitorização da cardiotoxicidade induzida por quimioterápicos encontra-se na população que, apesar da queda na FEVE, permanece dentro da faixa de normalidade de tal parâmetro. Por essas razões, existe o interesse de encontrar outros parâmetros ecocardiográficos e até mesmo biomarcadores que possam detectar uma disfunção miocárdica subclínica precedendo a queda da FEVE para uma faixa considerada anormal, se comparada às referências atuais. ${ }^{19}$

Nesse cenário, surgiram as pesquisas envolvendo outros parâmetros ecocardiográficos (análise da função diastólica e o strain/strain rate) e os biomarcadores (troponina, peptídeo atrial natriurético $(\mathrm{BNP})$, proteína $\mathrm{C}$ reativa $(\mathrm{PCR})$ e interleucina-ST2. ${ }^{20}$

Os parâmetros diastólicos têm demonstrado ser marcadores alternativos mais sensíveis que a FEVE para detectar disfunção cardíaca sutil em muitas patologias, particularmente na isquemia. ${ }^{19}$ Alguns estudos recentes reportam variações nesses parâmetros, como na relação E/A, E/e' e no índice de performance miocárdico ao comparar indivíduos normais e adultos em tratamento com antracíclicos. ${ }^{21}$

Contudo, essas variações nos parâmetros diastólicos estão, na maior parte das vezes, dentro do intervalo considerado normal e apresentaram uma correlação pobre entre as anormalidades diastólicas pós-quimioterapia e a redução da fração de ejeção do ventrículo esquerdo. ${ }^{22} \mathrm{O}$ valor das anormalidades diastólicas em predizer o desenvolvimento de disfunção sistólica ventricular em 3 meses foi sugerido em três pequenos estudos, ${ }^{23-26}$ entretanto, nenhum desses estudos demonstrou conclusivamente o valor prognóstico dos parâmetros diastólicos em predizer a cardiotoxicidade tardia. Assim, o papel dos parâmetros diastólicos no diagnóstico de cardiotoxicidade permanece controverso. ${ }^{10,27}$

Novas técnicas ecocardiográficas, como o Doppler tecidual, a análise da deformação tecidual (strain e strain rate) e variantes, têm sido agregadas, fornecendo subsídios para detecção precoce da disfunção ventricular esquerda em pacientes tratados com quimioterapia.

A análise do Doppler tecidual (DTI) mostrou-se menos sensível às alterações de pré-carga e à frequência cardíaca, em comparação aos parâmetros diastólicos e, por isso, estudos demonstraram sua utilidade, incluindo a medida da velocidade sistólica endocárdica (onda S') e a taxa de deformação miocárdica, na detecção precoce de cardiotoxicidade induzida pela doxorrubicina e pelo trastuzumabe, precedendo a queda da FEVE em 3 meses, no grupo com cardiotoxicidade. ${ }^{28,29}$

Ainda nesse contexto, strain e strain rate são índices ecocardiográficos de deformação miocárdica regional e global. O strain é definido como a mudança fracional no comprimento de um segmento do miocárdio em relação ao comprimento na diástole final e é normalmente expresso em porcentagem (\%). Por convenção, um valor positivo do strain reflete o alongamento do item analisado quando comparado ao seu tamanho original; enquanto um valor negativo está relacionado ao encurtamento do tamanho do objeto. ${ }^{30,31}$ Strain e strain rate interrelacionam-se pela derivação temporal da mesma forma que a velocidade e a distância. ${ }^{31}$

O strain (deformação) do ventrículo esquerdo pode ser avaliado pelo Doppler tecidual (DTI). Esta técnica, entretanto, é limitada à direção do feixe de ultrassom e, normalmente, é realizada apenas na direção longitudinal ou, em poucos segmentos selecionados, nas direções radial ou circunferencial. O método é demorado, sofre influência do ângulo, tem alta variabilidade intra e interobservador, além de requerer protocolos de imagem específicos. Isso tem limitado o seu uso na prática clínica, apesar de ainda utilizado em estudos de investigação. ${ }^{32}$

Para eliminar o problema da dependência de ângulo na análise do strain pelo Doppler tecidual, foi desenvolvida a técnica de aferição do strain baseada no rastreamento de pontos (speckle tracking) pela ecocardiografia bidimensional (2D). ${ }^{33}$ Nela, marcadores acústicos naturais, denominados speckles (os pontos brancos visualizados nas imagens em escala de cinza na ecocardiografia bidimensional), representam padrões específicos do tecido no miocárdico - como uma impressão digital daquele segmento. O software de análise do speckle tracking pelo eco 2D identifica os speckles (pontos com características únicas) e rastreia os seus movimentos em todas as direções, sendo o strain (deformação do miocárdio) avaliado com base na comparação dos padrões, quadro a quadro. $^{34}$ 
O strain é calculado para cada segmento do ventrículo esquerdo como a média relativa da deformação entre dois pontos ao longo das direções pré-definidas do sistema de coordenadas do coração: direções circunferencial, longitudinal e radial. ${ }^{9}$ Valores normais para o strain longitudinal estão na faixa entre -18 e - $22 \%$, diferindo ligeiramente dos algoritmos de análise do speckle tracking dos diferentes fabricantes. ${ }^{35,36} \mathrm{O}$ strain e o strain rate podem ser avaliados em cada região do ventrículo estudado (strain regional) e a média destes valores passa a representar o strain global, o que reflete a função global ventricular. ${ }^{37}$

A deformação miocárdica (strain) e a taxa de deformação miocárdica (strain rate) oferecem uma abordagem, aparentemente, mais sensível que a fração de ejeção do ventrículo esquerdo para mensurar cardiotoxicidade. Essas medidas podem fornecer uma avaliação multidimensional da mecânica miocárdica (longitudinal, radial e circunferencial), com a vantagem adicional de ser capaz de detectar movimentos anormais, sutis, da função regional da parede ventricular, o que não leva à diminuição global da FEVE. ${ }^{38,39}$

Enquanto alguns estudos reportam à queda no strain radial e circunferencial, a maioria dos estudos agora reportam exclusivamente ao strain longitudinal devido à significante variabilidade vista nos outros tipos de strain, principalmente no strain radial. ${ }^{9}$

Uma revisão sistemática publicada em 2014 revisou vários pequenos estudos clínicos para analisar a utilidade do strain no cenário da cardiotoxicidade. Nessa revisão, dados relativos a 1504 pacientes foram revisados e demonstraram que, em todos os estudos, a queda do strain foi preditiva de cardiomiopatia induzida por quimioterápicos. ${ }^{40} \mathrm{O}$ parâmetro mais eficiente em demonstrar o declínio na FEVE ou a insuficiência cardíaca foi, durante a análise, a queda de 10 a $15 \%$ no strain global longitudinal (speckle tracking). Tanto o strain global radial quanto o circunferencial também mostraram-se consistentemente anormais nesses pacientes, embora menos eficientes em demonstrar desfechos cardíacos. ${ }^{25}$

Em relação ao valor prognóstico dos índices de deformação miocárdicos, os dados permanecem limitados e o tempo de seguimento desses pacientes permanece incerto, uma vez que os efeitos tóxicos dos quimioterápicos são de longo prazo. ${ }^{41-43}$

As troponinas I e T são proteínas regulatórias cardíacas que controlam a interação actina-miosina mediada pelo cálcio. A troponina I é um marcador de injúria miocárdica, com liberação proporcional à extensão do dano cardíaco. ${ }^{44}$

A despeito de classicamente ser utilizada para avaliação de dano miocárdico secundário a isquemia, já foi demonstrado em vários estudos que a elevação da troponina em pacientes submetidos à quimioterapia não está relacionada à injúria isquêmica, mostrando a correlação do marcador com dano ao cardiomiócito produzido pelas drogas. A troponina pode permanecer persistentemente elevada até um mês após o término da quimioterapia. ${ }^{45}$

Um estudo realizado por Cardinale e colaboradoes mostrou que um terço dos pacientes que receberam altas doses de quimioterapia apresentaram elevação de troponina em algum ponto durante o tratamento. Tal elevação mostrou-se como um preditor confiável de subsequente disfunção cardíaca persistente no seguimento de 7 meses, julgando-se pela queda na FEVE. ${ }^{26}$

Em outro estudo do mesmo autor, novamente a elevação da troponina foi associada a uma incidência maior de eventos cardíacos em um seguimento médio de 20 meses. A incidência de eventos cardíacos foi particularmente alta no grupo com elevação persistente da troponina, até 1 mês após a quimioterapia. ${ }^{25}$

Outros estudiosos, Sawaya e colaboradores, também investigaram o papel da combinação entre troponina ultrassensível e do GLS $<-19 \%$ como fatores preditivos para subsequente queda da fração de ejeção e insuficiência cardíaca com resultados positivos. ${ }^{7}$

Biomarcadores alternativos foram analisados nesse contexto, incluindo BNP, proteína $\mathrm{C}$ reativa e interleucina ST2, porém mostraram resultados conflitantes, não sendo, até o momento, considerados marcadores confiáveis para predizer a lesão miocárdica causada por quimioterápicos. ${ }^{7,27-29}$

\section{PREVENÇÃO E TRATAMENTO PRECOCE}

A prevenção da insuficiência cardíaca secundária à quimioterapia pode, teoricamente, ser conseguida através de medidas preventivas primárias em todos pacientes submetidos à quimioterapia ou, alternativamente, por meio da identificação precoce e da intervenção terapêutica em pacientes que são de risco ou estão em uma fase precoce reversível do processo da doença. ${ }^{5}$

Avaliando a prevenção primária, mudanças na administração da quimioterapia, no que diz respeito à droga, dose e método de administração, têm sido estudados. Existem evidências que demonstram uma menor cardiotoxicidade com o uso da formulação lipossomal dos antracíclicos, o que parece evitar a difusão do agente cardiotóxico através da parede dos vasos cardíacos, enquanto continua a permitir a penetração através da vasculatura neoplásica anormal. A administração dos antracíclicos em doses divididas, ao invés de bolus, também tem demonstrado reduzir a cardiotoxicidade, sem comprometer a eficácia da droga. ${ }^{10,44-48}$

A utilização de terapias cardioprotetoras adicionais também tem sido investigada. O dexrazoxane tem uma ação cardioprotetora: esta droga atua no metabolismo oxidativo por ação quelante ao íon ferro, impedindo a ação direta dos antracíclicos nas organelas intracelulares do cardiomiócito, a fim de inibir a produção e liberação de radicais livres responsáveis pelos mecanismos de apoptose celular. ${ }^{8}$

Uma revisão publicada no Cochrane mostrou que o dexrazoxane parece ser eficaz na redução da cardiotoxicidade resultante da terapia com antracíclicos. Houve alguma preocupação em relação ao potencial risco de aumento de malignidades secundárias, assim como uma possível diminuição na taxa de resposta ao tratamento em doentes que utilizaram o cardioprotetor. No entanto, a meta-análise não 
demonstrou diferença significativa na taxa de resposta ou sobrevida global neste grupo de tratamento. ${ }^{46}$

O uso da terapêutica padrão para insuficiência cardíaca (inibidores da enzima da conversão da angiotensina, beta bloqueadores e estatinas) na prevenção primária da cardiotoxicidade induzida por quimioterapia também tem sido avaliado: os estudos realizados sugerem uma tendência cardioprotetora dessas drogas, mas não foram alcançados resultados significantes. O tempo de seguimento desses ensaios foi curto, durando 6 meses ou menos; assim, o papel potencialmente cardioprotetor dessas drogas na prevenção da toxicidade cardíaca a longo prazo ainda não foi demonstrado. ${ }^{10,49}$

Por outro lado, os pacientes com disfunção de ventrículo esquerdo, estabelecida secundária à quimioterapia, são classificados como estágio B de insuficiência cardíaca pela classificação proposta pela American College of Cardiology
(ACC)/American Heart Association (AHA) e, portanto, deve ser iniciada a terapêutica padrão no tratamento da insuficiência cardíaca em tais casos.

Como discutido ao longo deste documento, os novos métodos de detecção precoce de disfunção do ventrículo esquerdo são interessantes para orientar o início da terapia adequada o mais cedo possível. Vários autores demonstraram que a queda na fração de ejeção, a diminuição do strain longitudinal global e a elevação das troponinas, quando guiaram o início precoce do tratamento da disfunção cardíaca, ajudaram na reversão da disfunção e evitaram o remodelamento das dimensões finais do ventrículo esquerdo. ${ }^{47-49}$

Com base na revisão exposta nesse documento segue abaixo o protocolo clínico para avaliação da cardiotoxicidade em pacientes submetidos à quimioterapia proposto pelo serviço de ecocardiografia do Hospital Universitário Walter Cantídio.

\section{Protocolo cardiotoxicidade induzida por quimioterápicos}

Admissão no protocolo:

Prontuário:

Nome:

Nascimento

Diagnóstico:

Data do diagnóstico:

Esquema quimioterápico:

Radioterapia: ( ) Sim N Não

ECG - Sempre calcular QTc

ECO 2D:

\begin{tabular}{|l|l|l|}
\hline \multicolumn{1}{|c|}{ Tempo } & FE (\%) & GLS (\%) \\
\hline Pré - QT & & \\
\hline 03 meses & & \\
\hline 06 meses & & \\
\hline 09 meses & & \\
\hline 12 meses & & \\
\hline
\end{tabular}

**Na impossibilidade de realização do strain global longitudinal (GLS) - especificar 
Troponina:

\begin{tabular}{|l|l|l|}
\hline \multicolumn{1}{|c|}{ Ciclo QT } & & Antes do próximo ciclo \\
\hline $1^{\circ}$ ciclo & & \\
\hline $2^{\circ}$ ciclo & & \\
\hline $3^{\circ}$ ciclo & & \\
\hline $4^{\circ}$ ciclo & & \\
\hline $5^{\circ}$ ciclo & & \\
\hline
\end{tabular}

\section{Conduta:}

1. Paciente previamente hipertenso:

- Assegurar que entre os anti-hipertensivos estejam inibidores da enzima de conversão da angiotensina (IECA) ou bloqueadores dos receptores da angiotensina (BRA) e beta bloqueadores (BB) se necessário.

2. Paciente com fração de ejeção do ventrículo esquerdo (FEVE) $>55 \%$ :

- Seguimento habitual com retorno ao cardiologista após a realização dos ecocardiogramas com strain previsto pelo protocolo.

3. Paciente com FEVE $\leq 55 \%$ e assintomático:

- Avaliar riscos e benefícios na escolha do esquema quimioterápico.

- $\quad$ Sem tratamento para disfunção ventricular: iniciar BB e IECA/BRA.

- Em tratamento para disfunção ventricular: manter tratamento e assegurar uso de BB e IECA/BRA.

- Consulta cardiológica mensal até o término da quimioterapia.

3.1. Após o término da quimioterapia:

- $\quad$ Se FEVE > 55\%: seguimento habitual.

- $\quad$ Se FEVE < 55\%: reavaliação a cada trimestre, no primeiro ano; a cada semestre, no segundo ano e anual, a partir do terceiro ano.

4. Alteração de troponina e/ou eco strain durante quimioterapia:

- Realizar eco strain e iniciar IECA/BRA associando, se necessário, doses mínimas de BB.

- Repetir troponina e eco strain em 4 semanas:

$\checkmark$ Se normalizou: continua quimioterapia e mantem medicações iniciadas.

$\checkmark$ Se não houve normalização: associar BB, repetir em 4 semanas troponina e eco strain.

* Se normalizou: continua quimioterapia e mantém medicações iniciadas.

* Se não houve normalização: otimizar medicações.

- Se houver queda no strain global longitudinal (GLS) entre 10-15\% do valor basal: discutir com oncologista sobre a manutenção/adaptação do esquema quimioterápico. 
5. Queda da FEVE durante a quimioterapia:

5.1. Queda FEVE $<10 \%$ do valor basal e paciente assintomático:

- Iniciar IECA/BRA e BB.

- Dosagem quinzenal de troponina.

- Repetir eco strain em 4 semanas:

$\checkmark$ Manutenção da FEVE ou melhora: prosseguir quimioterapia e seguimento cardiológico mensal.

- Discutir com oncologista sobre a manutenção/adaptação do esquema quimioterápico.

5.2. Queda da FEVE $>10 \%$ do valor basal, ou FEVE após o início da quimioterapia $<45 \%$, ou paciente sintomático:

- Iniciar ou otimizar tratamento com IECA/BRA e BB.

- Dosagem quinzenal de troponina.

- $\quad$ Repetir eco strain em 2 semanas.

- Discutir com oncologista sobre a manutenção/adaptação do esquema quimioterápico.

5.3. Após 2 semanas:

- $\quad$ FEVE $>45 \%$ :

$\checkmark \quad$ Manter tratamento para disfunção.

$\checkmark \quad$ Manter quimioterapia.

$\checkmark$ Reavaliar em 2 semanas.

- $\quad \mathrm{FEVE}<45 \%$ e assintomático:

$\checkmark$ Prosseguir tratamento para disfunção.

$\checkmark$ Discutir com oncologista sobre a manutenção/adaptação do esquema quimioterápico.

$\checkmark \quad$ Reavaliar em 2 semanas.

- Queda da FEVE ou paciente sintomático:

$\checkmark$ Manter tratamento para disfunção.

$\checkmark$ Discutir com oncologista sobre a manutenção/adaptação do esquema quimioterápico.

5.4. Seguimento após 2 semanas de reavaliação da quimioterapia:

- Melhora ou manutenção da FEVE:

$\checkmark \quad$ Reavaliar em 4 semanas e manter otimização para disfunção.

- Piora da FEVE:

$\checkmark$ Otimizar medicação e discutir com oncologista sobre a manutenção/adaptação do esquema quimioterápico.

$\checkmark \quad$ Reavaliar em 2 semanas.

$\checkmark$ Se paciente em classe funcional IV (NYHA) $\rightarrow$ internação hospitalar. 


\section{REFERÊNCIAS}

1. Tan TC, Scherrer-Crosbie M. Cardiac complications of chemotherapy: role of imaging. Curr Treat Options Cardiovasc Med. 2014;16(4):296

2. Lefrak EA, Pitha J, Rosenheim S, Gottlieb JA. A clinicopathologic analysis of adriamycin cardiotoxicity. Cancer. 1973;32(2):302-14.

3. Kalil Filho R, Hajjar LA, Bacal F, Hoff PM, Diz M del P, Galas FRBG, et al. I Diretriz Brasileira de Cardio-Oncologia da Sociedade Brasileira de Cardiologia. Arq Bras Cardiol. 2011;96(2 supl 1):1-52.

4. Rocha RM, Schneider RS, Moreira I. Cardio-oncologia - onde estamos? Revista Hospital Universitário Pedro Ernesto. 2013:12(Supl 1):93-9.

5. Albini A, Pennesi G, Donatelli F, Cammarota R, De Flora S, Noonan DM. Cardiotoxicity of anticancer drugs: the need for cardiooncology and cardio-oncological prevention. J Natl Cancer Inst. 2010;102(1):14-25.

6. O'Hare M, Murphy K, Mookadam F, Sharma A, Lee H. Cardiooncology part I: chemotherapy and cardiovascular toxicity. Expert Rev Cardiovasc Ther. 2015;13(5):511-8.

7. Sawaya H, Sebag IA, Plana JC, Januzzi JL, Ky B, Tan TC, et al. Assessment of echocardiography and biomarkers for the extended prediction of cardiotoxicity in patients treated with anthracyclines, taxanes, and trastuzumab. Circ Cardiovasc Imaging. 2012;5(5):596-603.

8. Cypriano AS. Diretriz assistencial para avaliação cardiológica do paciente oncológico [Internet]. São Paulo: Albert Einstein; 2015 [acesso em: 20 abr 2016]. Disponível em: https://www.google. com.br/url? sa $=$ t\&rct $=\mathrm{j} \& \mathrm{q}=\&$ esrc $=$ s\&source $=$ web $\& \mathrm{~cd}=1 \& \mathrm{ved}=$ 0ahUKEwju2OuD5MTTAhWDi5AKHTwAAssQFggmMAA\& url=https $\% 3 \mathrm{~A} \% 2 \mathrm{~F} \% 2 \mathrm{Fpubdiretrizes}$.einstein.br\%2Fdownload. aspx\%3FID\%3D\%257B85EEDD74-7D3F-41E1-BBDA-3BB81115 1155\%257D\&usg=AFQjCNGJGm45laGufX631XW7DG16cwTOT A\&cad $=$ rja

9. Almeida AL, Gjesdal O, Mewton N, Choi EY, Teixido-Tura G, Yoneyama K, et al. Speckle-Tracking para Ecocardiografia bidimensional - aplicações clínicas. Rev Bras Ecocardiogr Imagem Cardiovasc. 2013;26(1):38-49.

10. O'Hare M, Murphy K, Mookadam F, Sharma A, Lee H. Cardiooncology part II: the monitoring, prevention, detection and treatment of chemotherapeutic cardiac toxicity. Expert Rev Cardiovasc Ther. 2015;13(5):519-27.

11. Dolci A, Dominici R, Cardinale D, Sandri MT, Panteghini M. Biochemical markers for prediction of chemotherapyinduced cardiotoxicity: systematic review of the literature and recommendations for use. Am J Clin Pathol. 2008;130(5):688-95.

12. Bocchi EA, Braga FG, Ferreira SM, Rohde LE, Oliveira WA, Almeida DR, et al. III Diretriz brasileira de insuficiência cardíaca crônica. Arq Bras Cardiol. 2009;93(1 supl 1):1-71.

13. Sengupta PP, Northfelt DW, Gentile F, Zamorano JL, Khandheria BK. Trastuzumab-induced cardiotoxicity: heart failure at the crossroads. Mayo Clin Proc. 2008;83(2):197-203.

14. Monsuez JJ, Charniot JC, Vignat N, Artigou JY. Cardiac sideeffects of cancer chemotherapy. Int J Cardiol. 2010;144(1):3-15.
15. Yeh ET, Bickford CL. Cardiovascular complications of cancer therapy: incidence, pathogenesis, diagnosis, and management. J Am Coll Cardiol. 2009;53(24):2231-47.

16. Wells QS, Lenihan DJ. Reversibility of left ventricular dysfunction resulting from chemotherapy: can this be expected? Prog Cardiovasc Dis. 2010;53(2):140-8.

17. Curtis JP, Sokol SI, Wang Y, Rathore SS, Ko DT, Jadbabaie F, et al. The association of left ventricular ejection fraction, mortality, and cause of death in stable outpatients with heart failure. J Am Coll Cardiol. 2003;42(4):736-42.

18. Ewer MS, Ali MK, Mackay B, Wallace S, Valdivieso M, Legha $\mathrm{SS}$, et al. A comparison of cardiac biopsy grades and ejection fraction estimations in patients receiving adriamycin. J Clin Oncol. 1984;2(2):112-17.

19. Moller JE, Pellikka PA, Hillis GS, Oh JK. Prognostic importance of diastolic function and filling pressure in patients with acute myocardial infarction. Circulation. 2006;114(5):438-44.

20. Mitani I, Jain D, Joska TM, Burtness B, Zaret BL. Doxorubicin cardiotoxicity: prevention of congestive heart failure with serial cardiac function monitoring with equilibrium radionuclide angiocardiography in the current era. J Nucl Cardiol. 2003;10(2):1329.

21. Dorup I, Levitt G, Sullivan I, Sorensen K. Prospective longitudinal assessment of late anthracycline cardiotoxicity after childhood cancer: the role of diastolic function. Heart. 2004;90(10):1214-16.

22. Tassan-Mangina S, Codorean D, Metivier M, Costa B, Himberlin C, Jouannaud C, et al. Tissue Doppler imaging and conventional echocardiography after anthracycline treatment in adults: early and late alterations of left ventricular function during a prospective study. Eur J Echocardiogr. 2006;7(2):141-6.

23. Stoddard MF, Seeger J, Liddell NE, Hadley TJ, Sullivan DM, Kupersmith J. Prolongation of isovolumetric relaxation time as assessed by Doppler echocardiography predicts doxorubicin-induced systolic dysfunction in humans. J Am Coll Cardiol. 1992;20(1):62-9.

24. Cardinale D, Colombo A, Torrisi R, Sandri MT, Civelli M, Salvatici M, et al. Trastuzumab-induced cardiotoxicity: clinical and prognostic implications of troponin I evaluation. J Clin Oncol. 2010;28(25):3910-6.

25. Cardinale D, Sandri MT, Colombo A, Boeri M, Lamantia G, Civelli M, et al. Prognostic value of troponin I in cardiac risk stratification of cancer patients undergoing high-dose chemotherapy. Circulation. 2004;109(22):2749-54.

26. Cardinale D, Sandri MT, Martinoni A, Tricca A, Civelli M, Lamantia $G$, et al. Left ventricular dysfunction predicted by early troponin I release after high-dose chemotherapy. J Am Coll Cardiol. 2000;36(2):517-22.

27. Daugaard G, Lassen U, Bie P, Pedersen EB, Jensen KT, Abildgaard $\mathrm{U}$, et al. Natriuretic peptides in the monitoring of anthracycline induced reduction in left ventricular ejection fraction. Eur J Heart Fail. 2005;7(1):87-93.

28. Dodos F, Halbsguth T, Erdmann E, Hoppe UC. Usefulness of 
myocardial performance index and biochemical markers for early detection of anthracycline-induced cardiotoxicity in adults. Clin Res Cardiol 2008;97(5):318-26.

29. Lenihan DJ, Massey MR, Baysinger KB, Adorno CL, Warneke $\mathrm{CL}$, Steinert D, et al. Superior detection of cardiotoxicity during chemotherapy using biomarkers. J Card Fail. 2007;13(6 Supl 2)):S151.

30. Mirsky I, Parmley WW. Assessment of passive elastic stiff $\neg$ ness for isolated heart muscle and the intact heart. Circ Res. $1973 ; 33(2): 233-43$.

31. Rademakers FE, Rogers WJ, Guier WH, Hutchins GM, Siu CO, Weis $\neg$ feldt ML, et al. Relation of regional cross-fiber shortening to wall thickening in the intact heart. Three-dimensional strain analysis by nmr tagging. Circulation. 1994;89(3):1174-82.

32. Helle-Valle T, Crosby J, Edvardsen T, Lyseggen E, Amundsen $\mathrm{BH}$, Smith HJ, et al. New noninvasive method for assessment of left ventricular rotation: speckle tracking echocardiography. Circula $\neg$ tion. 2005;112(20):3149-56.

33. Ishizu T, Seo Y, Enomoto Y, Sugimori H, Yamamoto M, Machino $\mathrm{T}$, et al. Experimental validation of left ventricular transmural strain gradient with echocardiographic two-dimensional speckle tracking imaging. Eur J Echocardiogr. 2010;11(4):377-85.

34. Korinek J, Wang J, Sengupta PP, Miyazaki C, Kjaergaard $\mathrm{J}$, Mc $\neg$ Mahon E, et al. Two-dimensional strain--a dopplerindepen $\neg$ dent ultrasound method for quantitation of regional defor $\neg$ mation: Validation in vitro and in vivo. J Am Soc Echocardiogr. 2005;18(12):1247-53.

35. Takigiku K, Takeuchi M, Izumi C, Yuda S, Sakata K, Ohte N, et al. Normal range of left ventricular 2-dimensional strain. Circ J. 2012;76(11):2623-32.

36. Manovel A, Dawson D, Smith B, Nihoyannopoulos P. Assessment of left ventricular function by different speckle-tracking softwa $\neg$ re. Eur J Echocardiogr. 2010;11(5):417-21.

37. Reisner SA, Lysyansky P, Agmon Y, Mutlak D, Lessick J, Friedman Z. Global longitudinal strain: A novel index of left ventricular systo $\neg$ lic function. J Am Soc Echocardiogr. 2004;17(6):630-3.

38. Jurcut R, Wildiers H, Ganame J, D’Hooge J, Paridaens R, Voigt JU. Detection and monitoring of cardiotoxicity-what does modern cardiology offer? Support Care Cancer. 2008;16(5):437-45.

39. Kowalski M, Kukulski T, Jamal F, D’Hooge J, Weidemann F, Rademakers F, et al. Can natural strain and strain rate quantify regional myocardial deformation? A study in healthy subjects. Ultrasound Med Biol. 2001;27(8):1087-97.

40. Thavendiranathan P, Poulin F, Lim KD, Plana JC, Woo A, Marwick TH. Use of myocardial strain imaging by echocardiography for the early detection of cardiotoxicity in patients during and after cancer chemotherapy: a systematic review. J Am Coll Cardiol. 2014;63(25 pt A):2751-68.

41. Seidman A, Hudis C, Pierri MK, Shak S, Paton V, Ashby M, et al. Cardiac dysfunction in the trastuzumab clinical trials experience. J Clin Oncol. 2002;20(5):1215-21.

42. Sá MP, Gomes RA, Silva NP, Sá MV, Calado I Filho. Cardiotoxicidade e Quimioterapia. Rev Bras Clin Med. 2009;7:32630 .

43. Zipes DP, Camm AJ, Borggrefe M, Buxton AE, Chaitman B, Fromer M, et al. ACC/AHA/ESC 2006 guidelines for management of patients with ventricular arrhythmias and the prevention of sudden cardiac death: a report of the American College of Cardiology/ American Heart Association Task Force and the European Society of Cardiology Committee for Practice Guidelines (Writing Committee to Develop Guidelines for Management of Patients With Ventricular Arrhythmias and the Prevention of Sudden Cardiac Death). J Am Coll Cardiol. 2006;48(5):e247-346.

44. Rivera E. Liposomal anthracyclines in metastatic breast cancer: clinical update. Oncologist. 2003;8(Supl 2):3-9.

45. Gabizon AA. Stealth liposomes and tumor targeting: one step further in the quest for the magic bullet. Clin Cancer Res. 2001;7(2):223-5.

46. van Dalen EC, Caron HN, Dickinson HO, Kremer LC. Cardioprotective interventions for cancer patients receiving anthracyclines. Cochrane Database Syst Rev. 2008;16(2):CD003917.

47. Cardinale D, Colombo A, Lamantia G, Colombo N, Civelli M, De Giacomi G, et al. Anthracycline-induced cardiomyopathy: clinical relevance and response to pharmacologic therapy. J Am Coll Cardiol. 2010;55(3):213-20.

48. Cardinale D, Colombo A, Sandri MT, Lamantia G, Colombo N, Civelli M, et al. Prevention of high-dose hemotherapy-induced cardiotoxicity in high-risk patients by angiotensin-converting enzyme inhibition. Circulation. 2006;114(23):2474-81.

49. Negishi K, Negishi T, Haluska BA, Hare JL, Plana JC, Marwick $\mathrm{TH}$, et al. Use of speckle strain to assess left ventricular responses to cardiotoxic chemotherapy and cardioprotection. Eur Heart J Cardiovasc Imaging. 2014;15(3):324-31.

\section{Como citar:}

Lima MB, Farias AG, Leopoldino DM. Protocolo do Hospital Universitário Walter Cantídio para avaliação de cardiotoxicidade em pacientes submetidos à quimioterapia. Rev Med UFC. 2018 abr-jun;58(2):75-85. 